\title{
A Unique Case of Mitral Valve Double Papillary Muscle Rupture
}

Luigi Pannone, MD, ${ }^{\text {a }}$ Giulio Falasconi, MD, ${ }^{\mathrm{a}}$ Alberto Margonato, MD, ${ }^{\mathrm{a}, \mathrm{b}}$ Elisabetta Lapenna, MD, ${ }^{\mathrm{c}}$ Roberto Spoladore, $\mathrm{MD}^{\mathrm{a}}$

\section{ABSTRACT}

Papillary muscle rupture is a rare and life-threatening complication of acute myocardial infarction (AMI). We present a unique case of double papillary muscle rupture in a patient with three vessel disease and acute thrombotic occlusion of left circumflex obtuse marginal artery as the culprit lesion. (Level of Difficulty: Beginner.) (J Am Coll Cardiol Case Rep 2019;1:428-30) (c) 2019 The Authors. Published by Elsevier on behalf of the American College of Cardiology Foundation. This is an open access article under the CC BY-NC-ND license (http://creativecommons.org/licenses/by-nc-nd/4.0/).

79-year-old man was admitted to our hospital emergency room suffering from dyspnea of recent onset. His past medical history was significant only for atrial fibrillation and hypertension. Electrocardiogram (Figure 1A) showed sinus tachycardia and inferior, posterior, and lateral ST-segment elevation myocardial infarction.

Transthoracic echocardiogram showed akinetic apical lateral and mid inferolateral wall segments. Left ventricular ejection fraction was mildly reduced (inspective ejection fraction 45\%). Color Doppler showed severe mitral regurgitation with bileaflet flail and double papillary muscle rupture. The patient underwent tracheal intubation, urgent coronary angiography, and intra-aortic-balloon pump (IABP) placement. Coronary angiogram showed 3-vessel disease with an acute thrombotic occlusion of the left circumflex obtuse marginal artery (OM1) (Figures 1B and 1C).

The patient was hypotensive despite high-dose inotropes and IABP and was emergently taken to the operating room. Intraoperative transesophageal echocardiography is shown in Figures 1D and 1E (Videos 1, 2, and 3). Bileaflet flail and papillary muscle head systolic displacement in the left atrium can be appreciated. Double coronary artery bypass grafting was performed with implantation of 2 saphenous vein grafts to the left anterior descending coronary artery and to the posterior interventricular branch of the right coronary artery. At left atriotomy, examination of mitral valve confirmed the rupture of 2 heads of the anterolateral papillary muscle and 1 head of the posterior papillary muscle (Figure 1F). The mitral valve was excised and replaced by St. Jude Epic N. 29 bioprosthesis (St. Jude Medical, St. Paul, Minnesota). At right atriotomy, the tricuspid annulus was dilated and Kay's annuloplasty was performed. Then, after 2 months of intensive care stay due to hemodynamic instability and perioperative infection of surgical sternal wound, the patient was discharged and transferred to cardiac rehabilitation unit, where the clinical course was uneventful.

Double papillary muscle rupture is a rare complication after AMI. Previous reports $(1,2)$ describe this event in patients with 3-vessel disease and acute right coronary artery occlusion and posterolateral AMI. This is the first report of double papillary muscle rupture following acute thrombotic occlusion of OM1 in 3-vessel disease. There was a codominance of the left and right coronary artery so that the posterior papillary muscle was perfused by both the right coronary artery and OM1, the last one also supplying anterolateral papillary muscle. 
Early diagnosis is mandatory. Transthoracic echocardiography performed in the emergency room should raise the suspect of papillary muscle rupture in the context of AMI when severe mitral regurgitation with flail is observed.

Management is challenging. Patients are usually hemodynamically unstable and anesthesiology support is needed for intubation and mechanical ventilation. The patient should undergo urgent coronary angiography and placement of a mechanical circulatory support in the cath lab should be considered. For the mechanical support IABP, percutaneous ventricular assist devices such as Impella (Abiomed, Danvers, Massachusetts) or TandemHeart (Cardiac Assist, Inc., Pittsburgh, Pennsylvania) can be considered depending on patient characteristics and local expertise. Once coronary anatomy is known, urgent coronary artery bypass grafting using saphenous vein grafts to reduce procedure time and mitral valve replacement can be performed.

FIGURE 1 Multi-Imaging of Mitral Valve Double Papillary Muscle Rupture
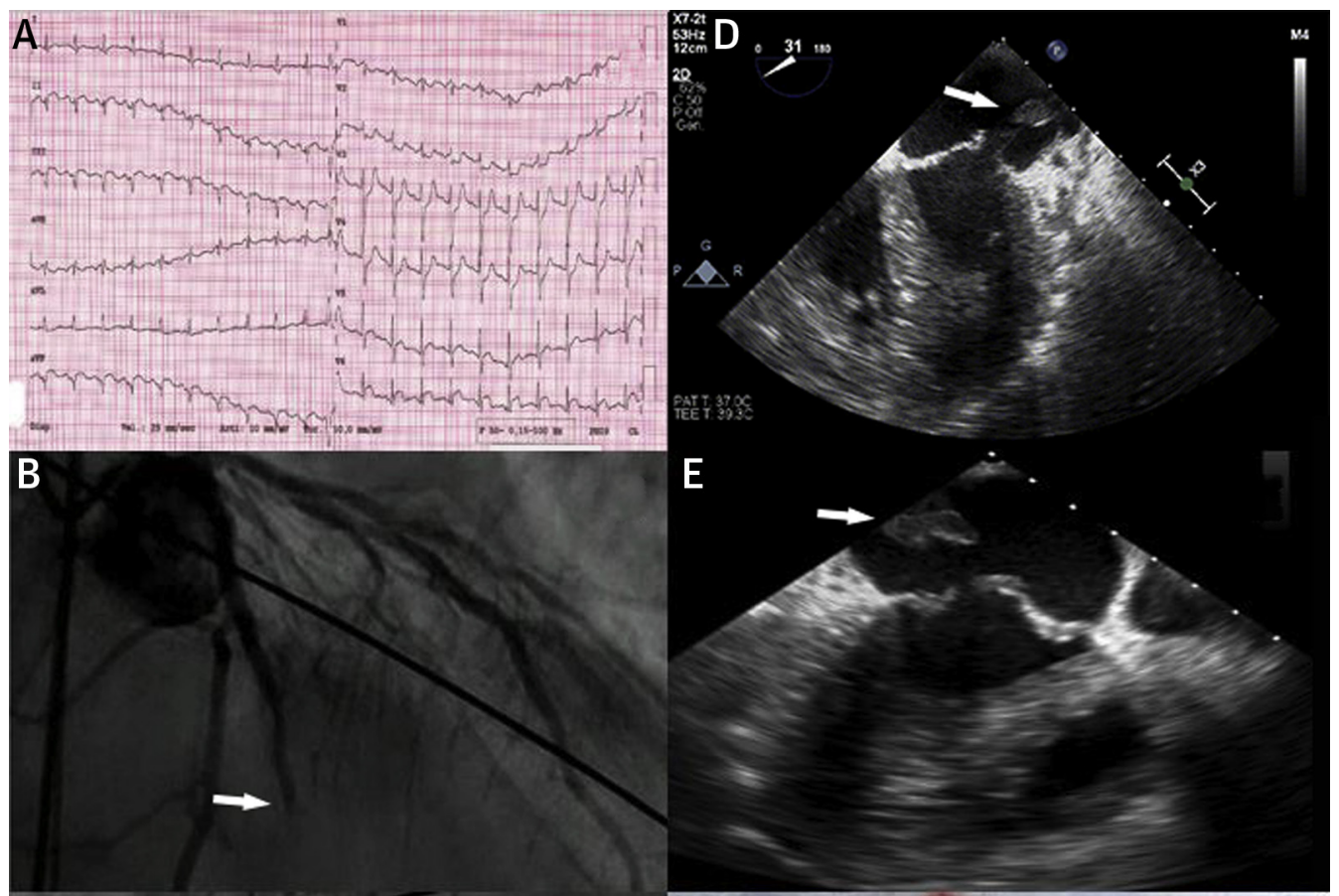

B
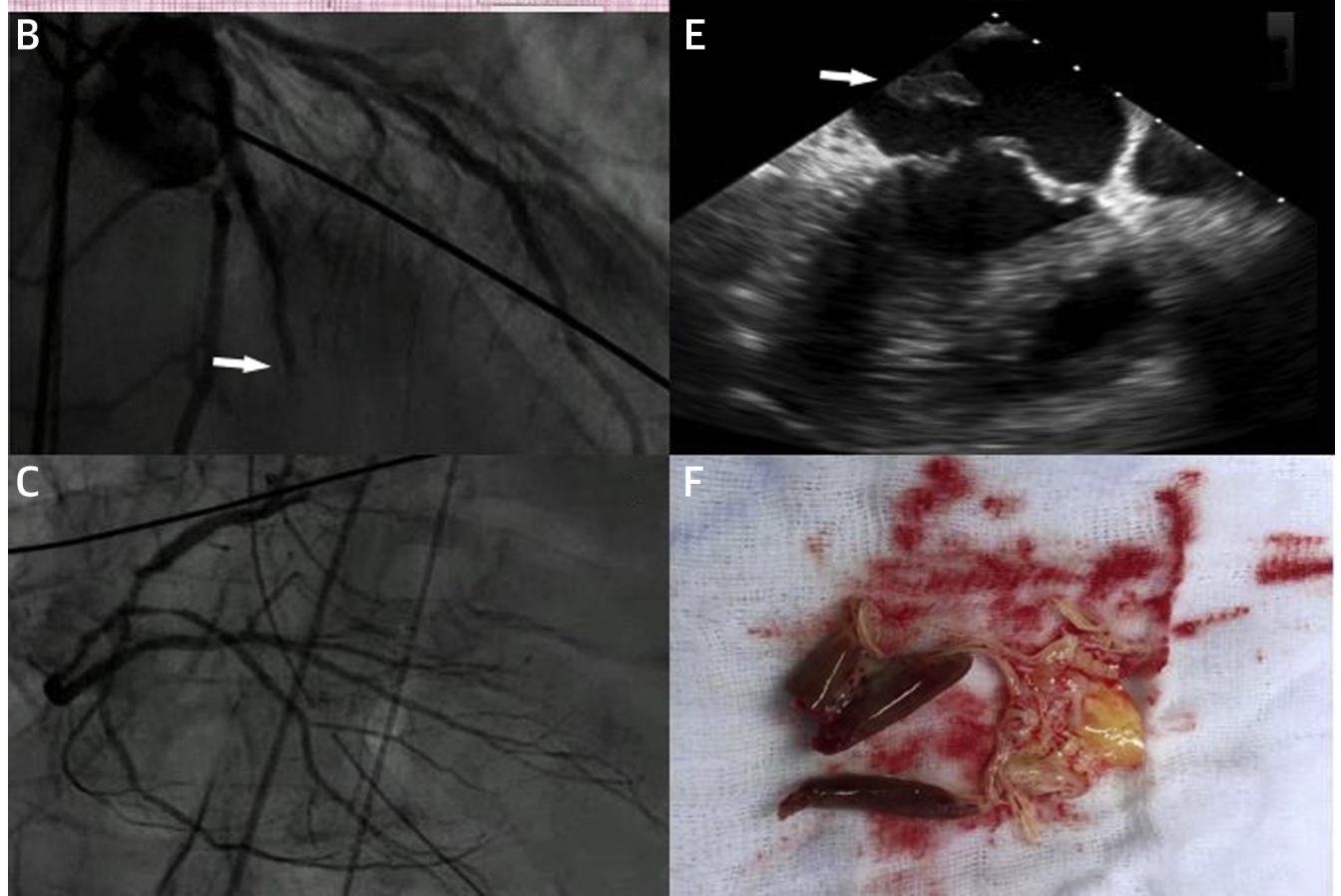

(A) Electrocardiogram. Sinus tachycardia and inferior, posterior, and lateral ST-segment elevation myocardial infarction. (B, C) Coronary angiogram. Three-vessel disease with an acute thrombotic occlusion of the left circumflex obtuse marginal artery (arrow). (D, E) Intraoperative transesophageal echocardiography. Bileaflet flail and papillary muscle heads systolic displacement in left atrium (Videos 1, 2, and 3). (F) Surgical specimen. Rupture of 2 heads of the anterolateral papillary muscle and 1 head of the posterior papillary muscle. 
ADDRESS FOR CORRESPONDENCE: Dr. Roberto Spoladore, Clinical Cardiology Unit, IRCCS San Raffaele University Hospital, Milan, Italy. E-mail: spoladore.roberto@hsr.it.

\section{REFEREN CES}

1. Lobo FV, King DEL, Heggtveit HA. Rupture of both left ventricular papillary muscles following acute myocardial infarction. Can J Cardiol 1990;6 66-70
2. Lobo FV, Fernandes J, Heggtveit HA. Postinfarction double papillary muscle rupture in three surgically excised mitral valves. Cardiovasc Patho 2005;14:251-5.
KEY WORDS echocardiography, myocardial infarction, papillary muscles

FIA APENDIX For supplemental videos, please see the online version of this paper. 